\title{
XÁC ĐỊNH HÀM LƯỢNG NICOTIN TRONG NƯỚC TIỂU BẰNG PHƯƠNG PHÁP CHIẾT LỎNG - RẮN VÀ GC/MS
}

\author{
PHẦ 2: XÁC ĐỊNH HÀM LƯợNG NICOTINE VÀ CÁC CHẤT \\ CHUYỀN HÓA
}

\author{
Trần Thị Thanh Vân ${ }^{1}$, Thành Thị Thu Thủy ${ }^{2, *}$ \\ ${ }^{I}$ Viện Nghiên cưu và Úng dụng Công nghệ Nha Trang, Viện Hàn lâm KHCNVN, \\ 2 Hùng Vuơng, Nha Trang \\ ${ }^{2}$ Viện Hóa học, Viện Hàn lâm KHCNVN, 18 Hoàng Quốc Việt, Cầu Giấy, Hà Nội \\ *Email: thuyttt@ich.vast.ac.vn
}

Đến Tòa soạn: 23/3/2013; Chấp nhận đăng: 27/8/2013

\section{TÓM TẮT}

Mục đích của chúng tôi là nghiên cứu quy trình phân tích định lượng nicotin trong nước tiểu bằng phương pháp chiết lỏng rắn và phương pháp sắc kí khí khối phổ (GC-MS). Trong phần 1 , chúng tôi đã báo cáo quy trình tách chiết nicotin từ nước tiểu bằng kỹ thuật chiết lỏng-rắn, pha rắn là octadecyl silica $(\mathrm{C} 18)$ và quy trình định lượng nicotine bằng phương pháp GC-MS. Trong phần 2 , chúng tôi báo cáo về kết quả phân tích định tính nicotine và các chất chuyển hóa chính của nó và áp dụng quy trình định lượng nicotine để phân tích 20 mẫu nước tiểu của những người không hút thuốc lá và 10 mẫu nước tiểu của những người hút thuốc lá ở nhà máy thuốc lá Khánh hòa. Kết quả phân tích cho thấy hàm lượng nicotine trong nước tiểu nằm trong khoảng $0,042 \mathrm{mg} / \mathrm{l}$ tới $2,074 \mathrm{mg} / \mathrm{l}$.

Tù khóa: nicotine, urine, chiết lỏng - rắn, GC/MS.

\section{MỞ ĐẦU}

Nicotine là một thành phần ancaloid chính được tìm thấy chủ yếu trong cây thuốc lá và có tên hóa học là 3-(1-methyl-2-pyrrolidinyl). Chúng có độc tính cao nên dễ gây nhiễm độc nghiêm trọng hoặc tử vong. Trong môi trường nicotine được phát tán từ khói thuốc lá do những người hút thuốc lá thải ra, từ nơi sản xuất các sản phẩm có chứa nicotine. Theo thống kê của các nhà nghiên cứu thì trong số công nhân tiếp xúc nghề nghiệp với nicotine có tỉ lệ ung thư phổi là $4 \%$, cao hơn rất nhiều so với công nhân làm việc trong môi trường không tiếp xúc với nicotine [1].

Do vậy khi muốn cảnh báo điều kiện an toàn lao động cần phải xét nghiệm hàm lượng nicotine trong các mẫu máu, nước tiểu.... Trong một vài thập kỉ trở lại đây đã có rất nhiều công trình nghiên cứu được công bố về phân tích định lượng nicotine trong các mẫu không khí, nước, máu và nước tiểu. Các phương pháp thông dụng được áp dụng là trắc quang, phổ hồng ngoại, sắc 
kí khí khối phổ (GC-MS), sắc kí lỏng khối phổ (LC-MS), thử enzym phóng xạ, thử Elisa ... [2 5].

Trong phần 1 , chúng tôi đã báo cáo điều kiện tối ưu để chiết tách nicotine từ nước tiểu với độ thu hồi là $73,91 \%$ và giới hạn phát hiện là $0,03 \mathrm{ppm}$ và đưa ra quy trình định lượng nicotine trong nước tiểu. Trong bài báo này, chúng tôi thông báo các kết quả về xác định nicotine và chất chuyển hóa chính của nó trong nước tiểu của 30 công nhân nhà máy thuốc lá Khánh hòa trong đó có 20 người không hút thuốc và 10 người hút thuốc bằng phương pháp GC-MS.

\section{THỰC NGHIẸM}

\subsection{Hóa chất}

Tất cả các hóa chất sử dụng trong nghiên cứu đều có độ tinh khiết siêu sạch của Merck và Prolabor. Mẫu nicotine chuẩn của Merck.

\subsection{Mẫu phân tích}

Mẫu nước tiểu 24 giờ được thu từ 30 người trong đó có 20 người hút thuốc lá và 10 người không hút thuốc lá của công nhân nhà máy thuốc lá Khánh hòa. Chiết tách nicotin và chất chuyển hóa chính từ nước tiểu dựa theo phương pháp chiết lỏng-rắn của Edward E.K. Baidoo và cộng sự [6]. Chi tiết về quy trình xử lí và chiết tách đã được công bố trong phần 1.

\subsection{Phương pháp GC/MS}

Máy sắc kí khí khối phổ GC/MS-6890N/5973Agilent (Mỹ). Sử dụng cột HP-5MS, chiều dài $30 \mathrm{~m}$, đường kính trong $0,25 \mathrm{mmm}$, bề dày pha tĩnh $0,25 \mu \mathrm{m}$.

Chế độ chạy tối ưu đã được xác định trong phần 1: Tốc độ khí mang Heli là 1,8 ml/phút, chương trình gradient nhiệt độ: $55^{\circ} \mathrm{C}$ giữ 2 phút, tăng $20^{\circ} \mathrm{C} /$ phút đến $215^{\circ} \mathrm{C}$ và chạy $280{ }^{\circ} \mathrm{C}$ trong 6 phút. Chạy theo chế độ SIM (Selected Ion Monitor).

- Khoảng tuyến tính: 0,05 - 10 ppm;

- Độ thu hồi: 73,91\%.

- Độ lặp lại: $\bar{X}=0,8989 \pm 0,0094$ ppm, $C V=1,05 \%$.

- Giới hạn phát hiện: 0,03 ppm.

\section{KẾT QUẢ VÀ THẢO LUẬN}

\subsection{Xác định định tính nicotine và chất chuyển hóa chính}

Nicotine $\left(\mathrm{C}_{10} \mathrm{H}_{14} \mathrm{~N}_{2}\right.$, trọng lượng phân tử: 162,23$)$ là một trong các chất có nhiều chuyển hoá, khi đi vào cơ thể sẽ được chuyển hoá nhanh thành nhiều sản phẩm chuyển hoá bền như: cotinine, trans-3'-hydroxycotinine, nornicotine, nicotine-N'-oxide, cotinine-N-oxide, cotinine$\mathrm{N}$-glucorinode. Trong đó sản phẩm chuyển hoá chính là cotinine, chiếm $70-80 \%$. Ngoài ra trong nước tiểu của những người hút thuốc lá còn có chất gây nghiện caffeine [6 - 8].

Sắc kí đồ của mẫu nicotine và các chất chuyển hóa chính của (hình 1) có 3 thành phần lớn nhất là nicotine (hình $2 a$ ), chất chuyển hóa chính của nó là cotinine (hình $2 b$ ) và chất gây nghiện 
caffeine (hình 2c).

Phổ GC/MS của mẫu nicotine chiết tách từ nước tiểu đưa ra trong hình 3 , có peak cơ bản tại $\mathrm{m} / \mathrm{z}=84$ và một số peak có cường độ trung bình và nhỏ tại $\mathrm{m} / \mathrm{z}=133,161,119$ và 42 , ngoài ra còn một số peak có cường độ rất nhỏ khác. Sự hình thành mảnh $\mathrm{m} / \mathrm{z}=84$ được Liguo Song và cộng sự [9] giải thích là do sự phá vỡ liên kết giữa vòng pyridine và vòng pyrroldine. Các mảnh có cường độ nhỏ hơn được hình thành là do sự bẻ gãy của các liên kết $\mathrm{C}-\mathrm{C}, \mathrm{C}-\mathrm{N}$ của vòng pyrrolidine. Dựa vào đó chúng tôi đưa ra sơ đồ phân mảnh của nicotine trên hình 4 .

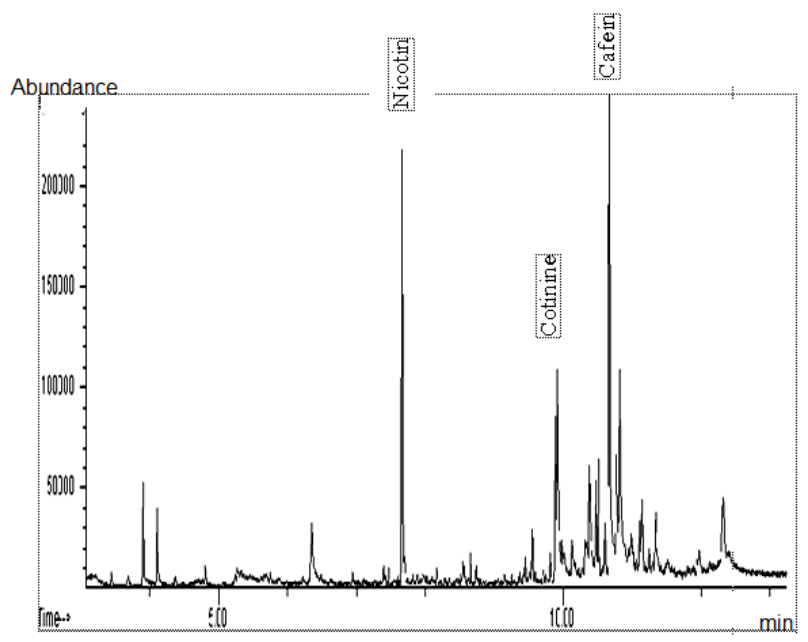

Hình 1. Sắc kí đồ của mẫu nicotine và các chất chuyển hóa chính trong mẫu nước tiểu.

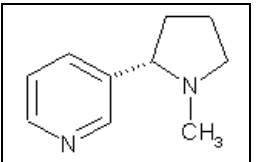

a)

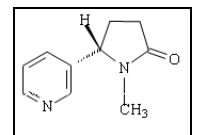

b)

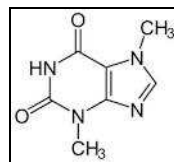

c)

Hình 2. Cấu trúc của nicotine a), cotinine b) và caffeine c).

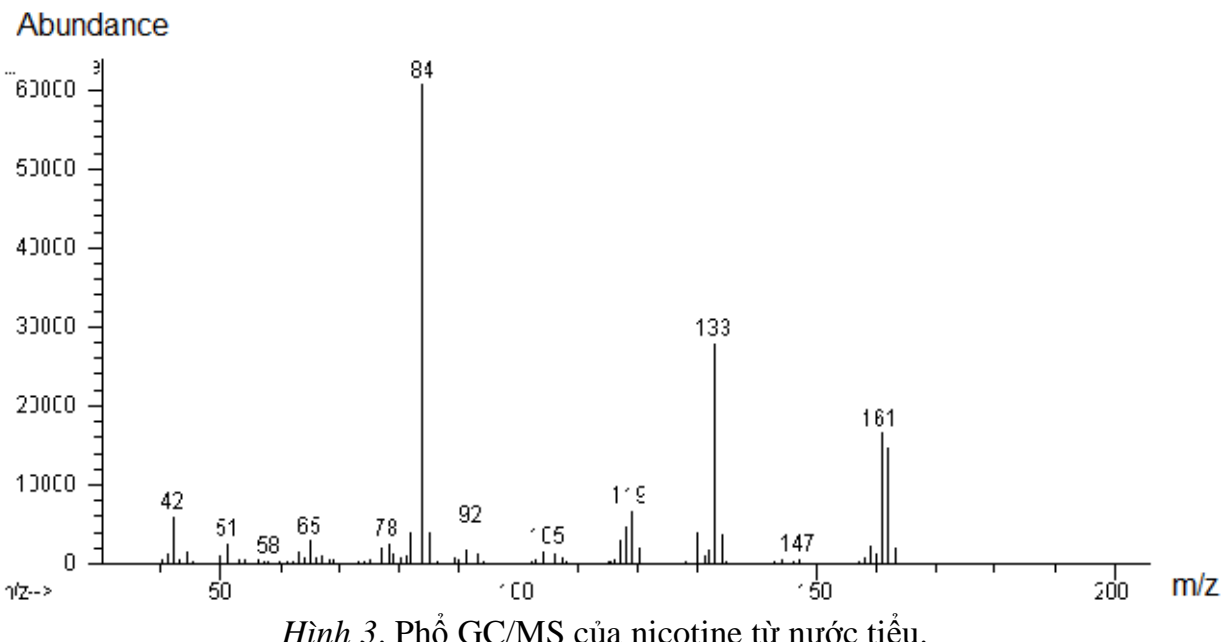


Trên sắc kí đồ của mẫu nicotine và các chất chuyển hóa ở hình 1 , còn có 2 tín hiệu có cường độ lớn, qua tham khảo tài liệu [6 - 8], đã được chúng tôi gán cho chất chuyển hóa chính cotinine $\left(\mathrm{C}_{10} \mathrm{H}_{12} \mathrm{~N}_{2} \mathrm{O}\right.$, trọng lượng phân tử 176,22) và chất gây nghiện caffeine $\left(\mathrm{C}_{8} \mathrm{H}_{10} \mathrm{~N}_{4} \mathrm{O}_{7}\right.$, trọng lượng phân tử 194,19). Điều này được khẳng định qua phổ $\mathrm{GC} / \mathrm{MS}$ của chúng (hình $5 \mathrm{a}$ và b).

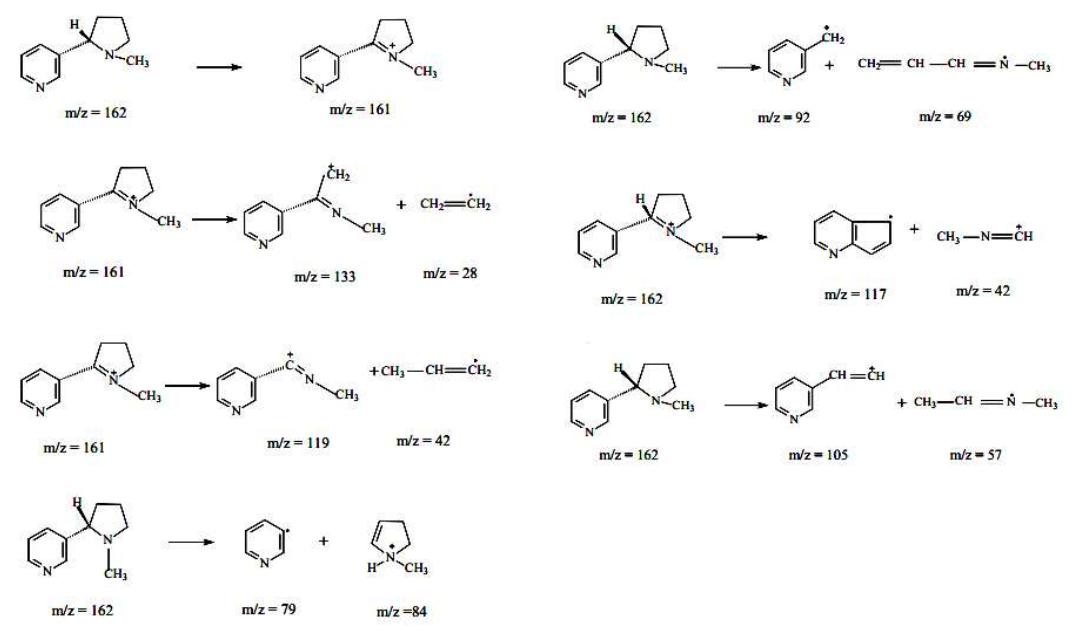

Hình 4. Sơ đồ phân mảnh của nicotine.
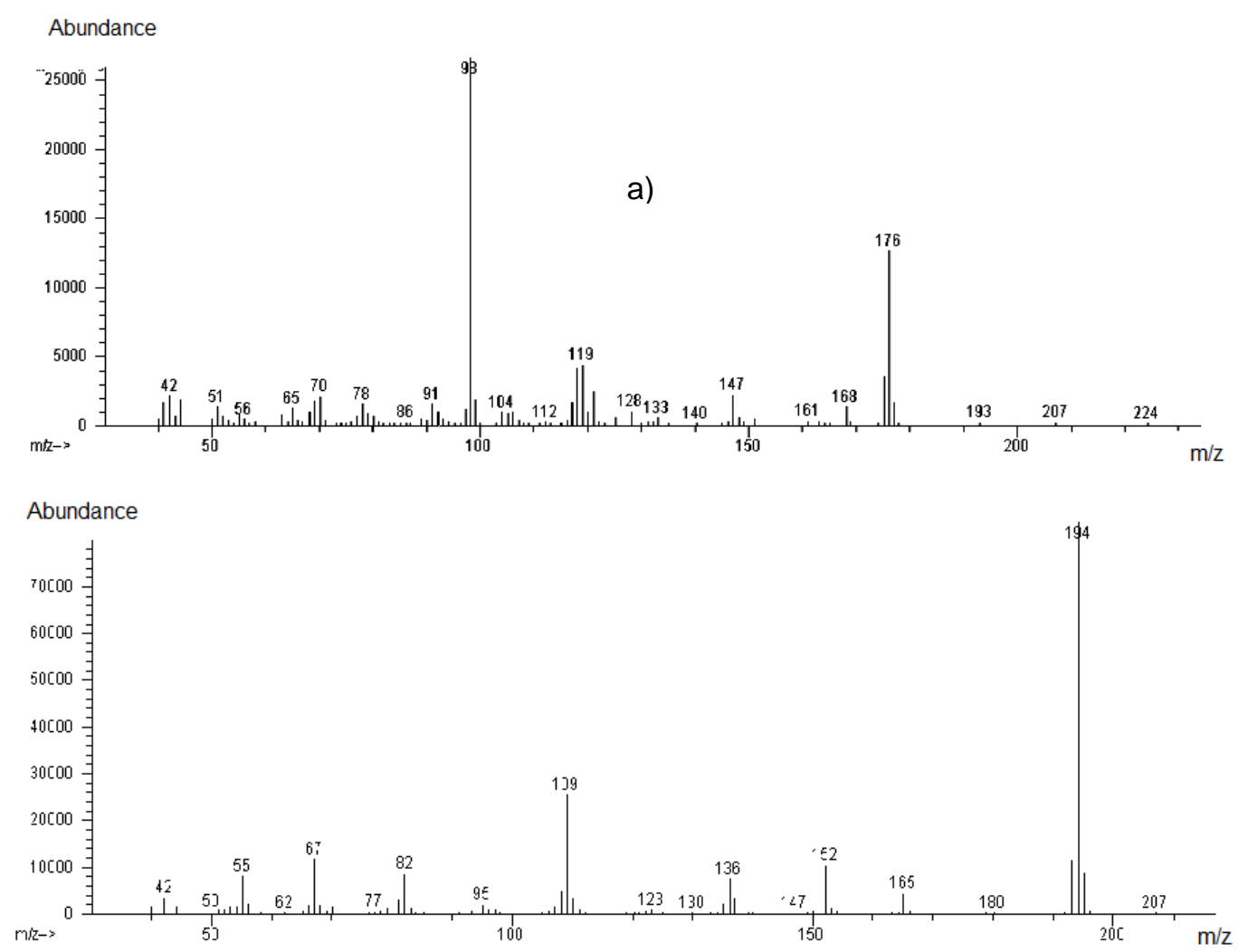

Hình 5. Phổ GC/MS của cotinine a) và caffeine b) trong mẫu nước tiểu. 


\subsection{Xác định hàm lượng nicotine trong mẫu nước tiểu}

Trong nghiên cứu định lượng nicotine, mảnh cơ bản thường được chọn làm mảnh định lượng, do vậy trong nghiên cứu này mảnh $\mathrm{m} / \mathrm{z} 84$ được chọn là mảnh định lượng. Từ phổ $\mathrm{GC} / \mathrm{MS}$ của nicotine chọn 5 mảnh đặc trưng là: $84,119,133,161,162$, các mảnh này không trùng lặp với các mảnh đặc trưng của các chất chuyển hóa của nicotine $[2,7,9,11]$. Để tăng độ nhạy khi phân tích định lượng chúng tôi dùng chế độ SIM (Selected Ion Monitor) để khảo sát nồng độ chất phân tích nicotine với mảnh định lượng là $\mathrm{m} / \mathrm{z} 84$ và các mảnh so sánh là $\mathrm{m} / \mathrm{z}=84,119,133,161,162$. Phổ GC/MS của mẫu nicotine chiết tách từ nước tiểu chạy theo chế độ SIM 5 mảnh đưa ra trên hình 6.

Sau đó chúng tôi tiếp tục khảo sát chế độ SIM 3 mảnh với mảnh định lượng 84 và các mảnh tham chiếu lần lượt là 119 và 133,119 và 161,119 và 162,133 và 161,133 và 162,161 và 162 và dựa vào kết quả này chúng tôi chọn phương án tối ưu là mảnh tham chiếu là 161 và 162 vì sắc kí đồ cho các peak không bị doãng vạch là điều kiện tốt cho phân tích định lượng.

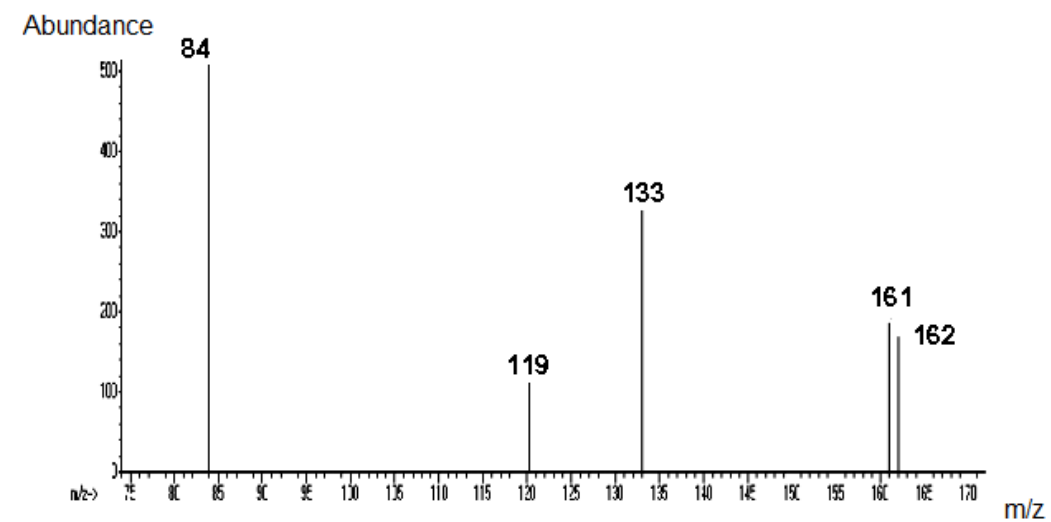

Hình 6. Phổ GC/MS của nicotine (0,1ppm) chạy chế độ SIM 5 mảnh.

Áp dụng quy trình chạy mẫu mà chúng tôi đã lựa chọn ở trên để phân tích 30 mẫu nước tiểu trong đó 20 mẫu là của người không hút thuốc lá và10 mẫu là của người hút thuốc lá của cán bộ nhà máy thuốc lá Khánh Hòa. Kết quả được đưa ra trên bảng 1.

Bảng 1. Kết quả phân tích hàm lượng nicotine trong mẫu nước tiểu (từ 1 - 20 là của người không hút thuốc lá và 21 - 30 là của người hút thuốc lá).

\begin{tabular}{|c|c|c|c|c|c|c|c|}
\hline \multirow{2}{*}{ STT } & \multicolumn{2}{|c|}{ Hàm lượng nicotine trong mẫu phân tích (mg/l) } & \multirow{2}{*}{ SD } & \multirow{2}{*}{ \% CV } & \multirow{2}{*}{ Ghi chú } \\
\cline { 2 - 7 } & Lần 1 & Lần 2 & Lần 3 & Trung bình & & & \\
\hline 1 & 0,0543 & 0,0568 & 0,0567 & 0,0559 & 0,0014 & 2,53 & \\
\hline $\mathbf{2}$ & $\mathbf{0 , 3 2 0 0}$ & $\mathbf{0 , 3 1 2 8}$ & $\mathbf{0 , 3 1 4 8}$ & $\mathbf{0 , 3 1 5 9}$ & $\mathbf{0 , 0 0 3 7}$ & $\mathbf{1 , 1 8}$ & $\begin{array}{l}\text { Vượt quá mức } \\
\text { cho phép }\end{array}$ \\
\hline 3 & 0,0580 & 0,0636 & 0,0580 & 0,0599 & 0,0032 & 5,40 & \\
\hline 4 & 0,1230 & 0,1179 & 0,1198 & 0,1202 & 0,0026 & 2,14 & \\
\hline 5 & 0,0680 & 0,0672 & 0,0724 & 0,0692 & 0,0028 & 4,05 & \\
\hline 6 & 0,0540 & 0,0517 & 0,0566 & 0,0541 & 0,0025 & 4,53 & \\
\hline
\end{tabular}


Trần Thị Thanh Vân, Thành Thị Thu Thủy

\begin{tabular}{|c|c|c|c|c|c|c|c|}
\hline 7 & 0,0420 & 0,0392 & 0,0427 & 0,0413 & 0,0019 & 4,48 & \\
\hline 8 & 0,4800 & 0,4799 & 0,4893 & 0,4831 & 0,0054 & 1,12 & $\begin{array}{l}\text { Vượt quá mức } \\
\text { cho phép }\end{array}$ \\
\hline 9 & 0,0630 & 0,0633 & 0,0587 & 0,0617 & 0,0026 & 4,17 & \\
\hline 10 & 0,2500 & 0,2515 & 0,2587 & 0,2534 & $\mathbf{0 , 0 0 4 7}$ & 1,84 & $\begin{array}{l}\text { Vượt quá mức } \\
\text { cho phép }\end{array}$ \\
\hline 11 & 0,1790 & 0,1792 & 0,1847 & 0,1810 & 0,0032 & 1,79 & \\
\hline 12 & 0,0450 & 0,0465 & 0,0450 & 0,0455 & 0,0009 & 1,90 & \\
\hline 13 & 0,0650 & 0,0631 & 0,0640 & 0,0640 & 0,0010 & 1,48 & \\
\hline 14 & 0,1980 & 0,1931 & 0,1904 & 0,1938 & 0,0039 & 1,99 & \\
\hline 15 & 0,1780 & 0,1805 & 0,1832 & 0,1806 & 0,0026 & 1,44 & \\
\hline 16 & 0,1980 & 0,2018 & 0,2024 & 0,2007 & 0,0024 & 1,19 & $\begin{array}{l}\text { Vượt quá mức } \\
\text { cho phép }\end{array}$ \\
\hline 17 & $\mathbf{0 , 3 6 0 0}$ & 0,3644 & 0,3648 & $\mathbf{0 , 3 6 3 1}$ & $\mathbf{0 , 0 0 2 7}$ & $\mathbf{0 , 7 3}$ & $\begin{array}{l}\text { Vượt quá mức } \\
\text { cho phép }\end{array}$ \\
\hline 18 & 0,0780 & 0,0747 & 0,0762 & 0,0763 & 0,0017 & 2,17 & \\
\hline 19 & 0,0980 & 0,0931 & 0,0948 & 0,0953 & 0,0025 & 2,61 & \\
\hline 20 & 0,6940 & 0,6971 & 0,7004 & 0,6972 & 0,0032 & 0,46 & \\
\hline 21 & 2,0740 & 2,0708 & 2,0679 & 2,0709 & $\mathbf{0 , 0 0 3 1}$ & 0,15 & $\begin{array}{l}\text { Vượt quá mức } \\
\text { cho phép }\end{array}$ \\
\hline 22 & 1,1500 & 1,1469 & 1,1427 & 1,1465 & 0,0037 & 0,32 & \\
\hline 23 & 0,4650 & 0,4662 & 0,4669 & 0,4660 & 0,0010 & 0,21 & \\
\hline 24 & 0,4012 & 0,4013 & 0,4087 & 0,4037 & 0,0043 & 1,07 & \\
\hline 25 & 0,9200 & 0,9271 & 0,9208 & 0,9226 & 0,0039 & 0,42 & \\
\hline 26 & 0,2600 & 0,2584 & 0,2640 & 0,2608 & 0,0029 & 1,11 & \\
\hline 27 & 0,4200 & 0,4154 & 0,4189 & 0,4181 & 0,0024 & 0,57 & \\
\hline 28 & 1,2850 & 1,2785 & 1,2727 & 1,2787 & 0,0062 & 0,48 & $\begin{array}{l}\text { Vượt quá mức } \\
\text { cho phép }\end{array}$ \\
\hline 29 & 1,3620 & 1,3575 & 1,2822 & 1,3339 & 0,0448 & 3,36 & $\begin{array}{l}\text { Vượt quá mức } \\
\text { cho phép }\end{array}$ \\
\hline 30 & 0,6150 & 0,6115 & 0,6111 & 0,6125 & 0,0021 & 0,35 & \\
\hline
\end{tabular}

Kết quả cho thấy tất cả các mẫu phân tích đều chứa nicotine và hàm lượng nicotine nằm trong dải rộng từ $0,042 \mathrm{ppm}$ đến $2,074 \mathrm{ppm}$. Theo TCVN $[12,13]$ để chuẩn đoán về bệnh nhiễm độc nicotine nghề nghiệp thì hàm lượng nicotine trong nước tiểu là $>0,3 \mathrm{mg} / 1$ (đối với người không hút thuốc) và $>1,2 \mathrm{mg} / \mathrm{l}$ (đối với người hút thuốc). So sánh với kết quả nhận được ở trên thì trong 30 mẫu phân tích có 4 mẫu $>0,3 \mathrm{mg} / 1$ (đối với người không hút thuốc) và 3 mẫu $>1,2$ $\mathrm{mg} / \mathrm{l}$ (đối với người hút thuốc). Kết quả phân tích này chứng tỏ rằng nicotine có mặt trong không khí đã xâm nhập vào cơ thể những cán bộ thường xuyên làm việc trong môi trường tiếp xúc với chúng. 


\section{KẾT LUẬN}

Với kĩ thuật chiết pha rắn và phương pháp GC/MS có thể chiết tách và xác định được đồng thời nicotine, chất chuyển hoá chính của nicotine là cotinine và chất gây nghiện cafein. Đã áp dụng quy trình trên để định lượng nicotine trong nước tiểu của 30 công nhân nhà máy thuốc lá Khánh hòa. Kết quả phân tích cho thấy hàm lượng nicotine trong nước tiểu nằm trong khoảng $0,042 \mathrm{mg} / \mathrm{l}$ tới $2,074 \mathrm{mg} / \mathrm{l}$. Điều đáng lưu ý là trong số 10 người không hút thuốc lá được điều tra, có tới 4 trường hợp có hàm lượng niotin trong nước tiểu vượt qua giới hạn cho phép theo TCVN. Tuy nhiên để có kết luận chắc chắn, cần mở rộng qui mô điều tra.

\section{TÀI LIỆU THAM KHẢO}

1. Hội nghị khoa học Y học lao động toàn quốc lần thứ IV, Báo cáo tóm tắt, 2001, tr. 17, 197.

2. Ho-Sang Shin, Jin-Gu Kim, Yoon-Jeong Shin, Sun Ha Jee - Sensitive and simple method for the determination of nicotine and cotinine in human urine, plasma and saliva by gas chromatography-mass spectrometry, Journal of Chromatography B $\mathbf{7 6 9}$ (2002) 177-183.

3. Allan S. Xu, Lana L. Pg, James A. Havel, Mary E. Petersen, John A. Fiene, James D. Hulse - Determination of nicotine and cotinine in human plasma by liquid chromatography-tandem mass spectrometry with atmospheric-pressure chemical ionazation interface, Journal of Chromatography B 682 (1996) 249-257.

4. Al-Tamrah S. A. - Spectrophotometric determination of nicotine, Analytica Chimica Acta 379 (1999) 75-80.

5. Baranowski J., Pochopien G., Baranowska I. - Determination of nicotine, cotinine and caffeine in meconium using high- performance liquid chromatography, Journal of Chromatography B 707 (1998) 317-321.

6. Edward E. K. Baidoo, Malcolm R. Clench, Robert F. Smith, Lee W. Tetler Determination of nicotine and its metabolites in urine by solid-phase extraction and sample stacking capillary electrophoresis-mass spectrometry, Journal of Chromatography B 796 (2003) 303-313.

7. Insook Kim, William D. Darwin, Marilyn A. Huestis - Simultaneous determination of nicotine, cotinine, norcotinine and trans-3'-hydroxyxotinine in humanoral fluid using solid phase extraction and gas chromatography-mass spectrometry, Journal of Chromatography B 814 (2005) 233-240.

8. Michael Meger, Irmtrud Meger-Kossien, Annette Schuler -Metz, Dinamis Janket, Gerhard Scherer - Simultaneous determination of nicotine and eight nicotine metabolites in urine of smokers using liquid chromatography-tandem mass spectrometry, Journal of Chromatography B 778 (2002) 251-261.

9. Liguo Song, Warren Davis, Sara M. Abrams, Jeremy Hemiup, A. Latif Kazim, K. Michael Cummings, Martin C. Mahoney - Sensitive and rapid method for the determination of urinary cotinine in non-smokers: an application for studies assessing exposures to second hand smoke (SHS), Analytica Chimica Acta 545 (2005) 200-208.

10. Byung Hwa Jung, Bong Chul Chung, Suk-Jae Chung, Min-Hwa Lee, Chang-Koo Shim Simultaneous GC-MS determination of nicotine and cotinine in plasma for the 
pharmacokinetic characterization of nicotine in rat, Journal of Pharmaceutical and Biomedical Analysis 20 (1999) 195-202.

11. Jin-gu Kim, Ueon-Sang Shin, Ho-Sang Shin - Rapid monitoring method of active and passive smoker with saliva cotinine by gas chromatography-mass spectrometry, Bull. Korean Chem. 23 (10) (2002) 1496-1498.

12. Viện $Y$ học lao động và vệ sinh môi trường - Thường quy kĩ thuật, Bộ Y tế, 1996.

13. Viện $\mathrm{Y}$ học lao động và Vệ sinh môi trường -21 bệnh nghề nghiệp được bảo hiểm, Bộ $\mathrm{Y}$ tế, 1997, tr. 399-416.

\title{
ABSTRACT \\ DETERMINATION OF NICOTIN IN HUMAN URINE BY USING SOLID-LIQUID EXTRACTION AND GC/MS.
}

\section{PART 2. DETERMINATION OF NICOTINE AND ITS METABOLITES}

\author{
Tran Thi Thanh Van ${ }^{1}$, Thanh Thi Thu Thuy ${ }^{2, *}$
}

Nha Trang Institute of Technology Research and Application, VAST, 2 Hung Vuong, Nha Trang

${ }^{2}$ Institute of Chemistry, VAST, 18 Hoang Quoc Viet, Cau Giay, Hanoi

*Email: thuyttt@ich.vast.ac.vn

The aim of our research is to determine the content of nicotine in human urine by using solid-liquid extraction technique and gas chromatography- mass spectrometry (GC-MS). In part 1 , the procedures of isolation of nicotine from urine by solid-liquid extraction and determination of nicotine by GC-MS were reported. In part 2, the results in determination of nicotine and its metabolite in urine samples taken from 20 non-smoking and 10 smoking volunteers will be reported. As a result, the content of nicotine in urine ranged from $0.042 \mathrm{mg} / \mathrm{l}$ to $2.074 \mathrm{mg} / \mathrm{l}$. It is worthy to note that four in ten non-smoking person showed nicotine content higher than Vietnam's standard.

Keywords: nicotine, urine, solid-liquid extraction, GC/MS. 